\title{
Nitrogen-deficient and iron-rich associated absorbers with oversolar metallicities towards the quasar HE0141-3932
}

\author{
S. A. Levshakov ${ }^{1}$, I. I. Agafonova ${ }^{1}$, D. Reimers ${ }^{2}$, C. Fechner ${ }^{2}$, \\ E. Janknecht ${ }^{2}$, and S. Lopez ${ }^{3}$ \\ ${ }^{1}$ A. F. Ioffe Physico-Technical Institute, 194021 St.Petersburg, Russia \\ email: lev@astro.ioffe.rssi.ru \\ ${ }^{2}$ Hamburger Sternwarte, Gojenbergsweg 112, 21029 Hamburg, Germany \\ ${ }^{3}$ Departamento de Astronomia, Universidad de Chile, Casilla 36-D, Santiago, Chile
}

\begin{abstract}
HE0141-3932 $\left(z_{\mathrm{em}}=1.80\right)$ is a bright blue radio-quiet quasar which reveals an emission line spectrum with an unusually weak $\operatorname{Ly} \alpha$ line. In addition, large redshift differences $(\Delta z=0.05)$ are observed between high ionization and low ionization emission lines. Absorption systems identified at $z_{\mathrm{abs}}=1.78,1.71$, and 1.68 show mild oversolar metallicities $\left(Z \approx 1-2 Z_{\odot}\right)$ and can be attributed to the associated gas clouds ejected from the circumnuclear region. The joint analysis of the emission and absorption lines leads to the conclusion that this quasar is seen almost pole-on. Its apparent luminosity may be Doppler boosted by $\sim 10$ times. The absorbing gas shows high abundance of $\mathrm{Fe}, \mathrm{Mg}$, and $\mathrm{Al}([\mathrm{Fe}, \mathrm{Mg}, \mathrm{Al} / \mathrm{C}] \simeq 0.15 \pm 0.10)$ along with underabundance of $\mathrm{N}([\mathrm{N} / \mathrm{C}] \leqslant-0.5)$. This abundance pattern is at variance with current chemical evolution models of QSOs predicting $[\mathrm{N} / \mathrm{C}] \gtrsim 0$ and $[\mathrm{Fe} / \mathrm{C}]<0$ at $Z \sim Z_{\odot}$. Full details of this work are given in Reimers et al. (2005).
\end{abstract}

Keywords. Cosmology: observations, line: formation, line: profiles, galaxies: abundances, quasars: absorption lines, quasars: individual: HE0141-3932

In the absorption spectrum of HE0141-3932 obtained with the VLT/UVES, associated systems are found at $z_{\text {abs }}=1.78,1.71$, and 1.68. They exhibit a wealth of metal absorptions including lines of FeII. Since these systems arise in gas ejected from the circumnuclear region, careful analysis of the element abundances can produce important clues about the enrichment mechanisms and physical processes leading to the QSO/AGN phenomena. The numbers presented in Table 1 are obtained with the Monte Carlo Inversion (MCI) method - an advanced computational procedure designed to estimate the physical parameters of optically thin absorbers with varying gas density and turbulent velocity field (Levshakov et al. 2000).

We conclude the following:

- The analyzed absorption systems show high iron content, $[\mathrm{Fe} / \mathrm{C}]=0.15 \pm 0.1$, $[\mathrm{Fe} / \mathrm{Mg}]=0.0 \pm 0.1\left(z_{\mathrm{abs}}=1.78\right)$, but at the same time nitrogen is strongly underabundant, $[\mathrm{N} / \mathrm{C}] \lesssim-0.5\left(z_{\mathrm{abs}}=1.68\right)$. These abundances are estimated in different associated systems but they are representative for the bulk of circumnuclear gas since the mass of the stellar population involved in the enrichment of quasar's circumnuclear region is high, $>10^{4} M_{\odot}$, (Baldwin et al. 2003) and, hence, large metallicity gradients and sharp discontinuities due to enrichment by only a few stars are unlikely.

- In the Hamann \& Ferland (1999) models of QSO's chemical evolution, solar metallicity is reached after $\gtrsim 0.2 \mathrm{Gyr}$ and is characterized by a relative overabundance of nitrogen, $[\mathrm{N} / \mathrm{C}] \gtrsim 0$, and an underabundance of iron, $[\mathrm{Fe} / \mathrm{C}]<0$. Due to delay of $1 \mathrm{Gyr}$ in Fe enrichment expected from longer evolution of SNe Ia which are the main source of 
Table 1. Metal abundances at $z_{\text {abs }}=1.7817,1.7103$, and 1.6838 towards HE0141-3932 derived by the MCI procedure (solar photospheric abundance for carbon is taken from Allende Prieto et al. (2002); for silicon, nitrogen and iron - from Holweger (2001))

\begin{tabular}{|c|c|c|c|c|c|}
\hline \multirow[b]{2}{*}{$\begin{array}{l}\text { Parameter } \\
(1)^{a}\end{array}$} & \multirow{2}{*}{$\begin{array}{c}z_{\mathrm{abs}}=1.7817 \\
(2)\end{array}$} & \multicolumn{2}{|c|}{$z_{\mathrm{abs}}=1.7103$} & \multicolumn{2}{|c|}{$z_{\mathrm{abs}}=1.6838$} \\
\hline & & $\begin{array}{l}\text { subsystem } A \\
(3)^{b}\end{array}$ & $\begin{array}{c}\text { subsystem } B \\
(4)^{b}\end{array}$ & $\begin{array}{c}\text { subsystem } A \\
(5)\end{array}$ & $\begin{array}{c}\text { subsystem } B \\
(6)\end{array}$ \\
\hline$\left[Z_{\mathrm{C}}\right]$ & $0.14 \pm 0.08$ & 0.65 & 0.94 & $\lesssim 0.33$ & $0.08 \pm 0.10$ \\
\hline$\left[Z_{\mathrm{N}}\right]$ & $<0.15$ & 0.56 & 0.58 & $\lesssim-0.3$ & $-0.48 \pm 0.15$ \\
\hline$\left[Z_{\mathrm{Mg}}\right]$ & $0.30 \pm 0.10$ & 0.6 & $\ldots$ & $\ldots$ & $\ldots$ \\
\hline$\left[Z_{\mathrm{Al}}\right]$ & $0.30 \pm 0.10$ & 0.3 & & $\ldots$ & $\ldots$ \\
\hline$\left[Z_{\mathrm{Si}}\right]$ & $0.09 \pm 0.08$ & 0.33 & 0.94 & $\lesssim 0.4$ & $0.14 \pm 0.10$ \\
\hline$\left[Z_{\mathrm{Fe}}\right]$ & $0.30 \pm 0.15$ & $\sim 1.5$ & $\ldots$ & $\ldots$ & $\ldots$ \\
\hline$N(\mathrm{H} \mathrm{I})$ & $(2.6 \pm 0.5) \mathrm{E} 15$ & $1.0 \mathrm{E} 16$ & $(2.6 \pm 0.5) \mathrm{E} 14$ & $(1.1 \pm 0.2) \mathrm{E} 14$ & $2.5 \mathrm{E} 15^{c}$ \\
\hline$N(\mathrm{C}$ II $)$ & $(1.64 \pm 0.08) \mathrm{E} 13$ & $(1.5 \pm 0.2) \mathrm{E} 14$ & $\lesssim 1.0 \mathrm{E} 12$ & $\lesssim 1.6 \mathrm{E} 11$ & $(7.3 \pm 1.5) \mathrm{E} 12$ \\
\hline$N\left(\mathrm{C}\right.$ II $\left.^{*}\right)$ & $<5.0 \mathrm{E} 11$ & $<4.3 \mathrm{E} 12$ & $\ldots$ & $\ldots$ & $<1.5 \mathrm{E} 12$ \\
\hline$N(\mathrm{Mg}$ II $)$ & $(1.20 \pm 0.20) \mathrm{E} 11$ & $(5.3 \pm 1.5) \mathrm{E} 12$ & $\cdots$ & $\ldots$ & $\ldots$ \\
\hline$N(\mathrm{Si}$ II $)$ & $(2.8 \pm 0.3) \mathrm{E} 12$ & $(1.2 \pm 0.1) \mathrm{E} 13$ & $\ldots$ & $\ldots$ & $\ldots$ \\
\hline$N\left(\mathrm{Si} \mathrm{II}^{*}\right)$ & $<1.3 \mathrm{E} 11$ & $<2.0 \mathrm{E} 11$ & $\ldots$ & $\ldots$ & $\ldots$ \\
\hline$N($ Fe II $)$ & $(1.4 \pm 0.4) \mathrm{E} 11$ & $(5.5 \pm 2.0) \mathrm{E} 11$ & $\ldots$ & $\ldots$ & $\ldots$ \\
\hline$N(\mathrm{Al}$ III $)$ & $(3.6 \pm 1.0) \mathrm{E} 11$ & $(1.4 \pm 0.3) \mathrm{E} 12$ & $\ldots$ & $\ldots$ & $\ldots$ \\
\hline$N(\mathrm{Si}$ III $)$ & $7.5 \mathrm{E} 12^{c}$ & $(5.5 \pm 0.6) \mathrm{E} 13$ & $(2.3 \pm 1.2) \mathrm{E} 11$ & $\lesssim 8.5 \mathrm{E} 10$ & $(7.9 \pm 0.8) \mathrm{E} 12$ \\
\hline$N(\mathrm{C}$ IV $)$ & $(2.2 \pm 0.2) \mathrm{E} 13$ & $(1.7 \pm 0.2) \mathrm{E} 15$ & $(3.4 \pm 0.3) \mathrm{E} 14$ & $(3.4 \pm 0.2) \mathrm{E} 13$ & $(2.3 \pm 0.1) \mathrm{E} 14$ \\
\hline$N(\mathrm{Si}$ IV $)$ & $(4.5 \pm 0.4) \mathrm{E} 12$ & $(7.7 \pm 0.8) \mathrm{E} 13$ & $(1.6 \pm 0.5) \mathrm{E} 12$ & $<3.0 \mathrm{E} 11$ & $(1.2 \pm 0.1) \mathrm{E} 13$ \\
\hline$N(\mathrm{~N} \mathrm{v})$ & $<1.4 \mathrm{E} 12$ & $(1.8 \pm 0.2) \mathrm{E} 14$ & $(7.7 \pm 0.8) \mathrm{E} 13$ & $(4.5 \pm 0.5) \mathrm{E} 12$ & $(1.3 \pm 0.1) \mathrm{E} 13$ \\
\hline
\end{tabular}

iron, the emission line ratios Fe II/C IV and Fe II/Mg II are proposed to be a measure of QSO ages. However, large values of $[\mathrm{Fe} / \mathrm{C}]$ and $[\mathrm{Fe} / \mathrm{Mg}]$ are always associated in these models with a considerable overabundance of nitrogen, $[\mathrm{N} / \mathrm{C}]>0.3$. We do not observe such relations in our systems and, hence, do not confirm this 'iron clock' model.

- The results obtained are in line with Matteucci \& Recchi (2001), who showed that time scale for enrichment by SNe Ia is not unique but a strong function of the adopted stellar lifetimes, initial mass function, and star formation rate and can vary by more than order of magnitude.

\section{Acknowledgements}

S.A.L. thanks the IAU for the travel grant.

\section{References}

Allende Prieto, C., Lambert, D.L., \& Asplund, M. 2002, ApJ 573, L137

Baldwin, J.A., Ferland, G.J., Korista, K.T., et al. 2003, ApJ 582, 590

Hamann, F., \& Ferland, G. 1999, ARA\&A 37, 487

Holweger, H. 2001, in Solar and Galactic Composition, ed. R. F. Wimmer-Schweingruber, AIP Conf. Proc. 598, 23

Levshakov, S.A., Agafonova, I.I., \& Kegel, W.H. 2000, A\&A 360, 833

Matteucci, F., \& Recchi, S. 2001, ApJ 558, 351

Reimers, D., Janknecht, E., Fechner, C., Agafonova, I.I., Levshakov, S.A., \& Lopez, S. 2005, $A \& A 435,17$ 


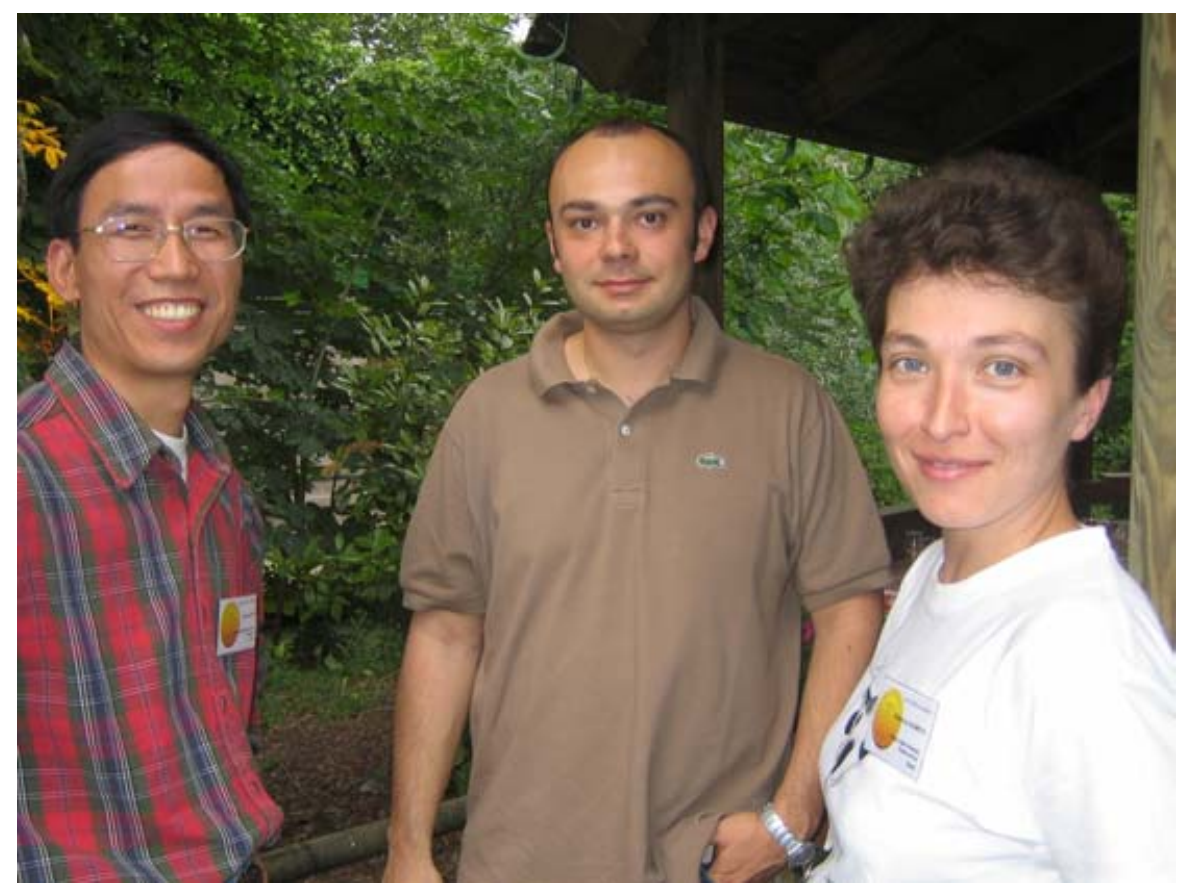

Jorge Mèlendez, Francesco Calura and Ekatarina Kasimova at the conference dinner.

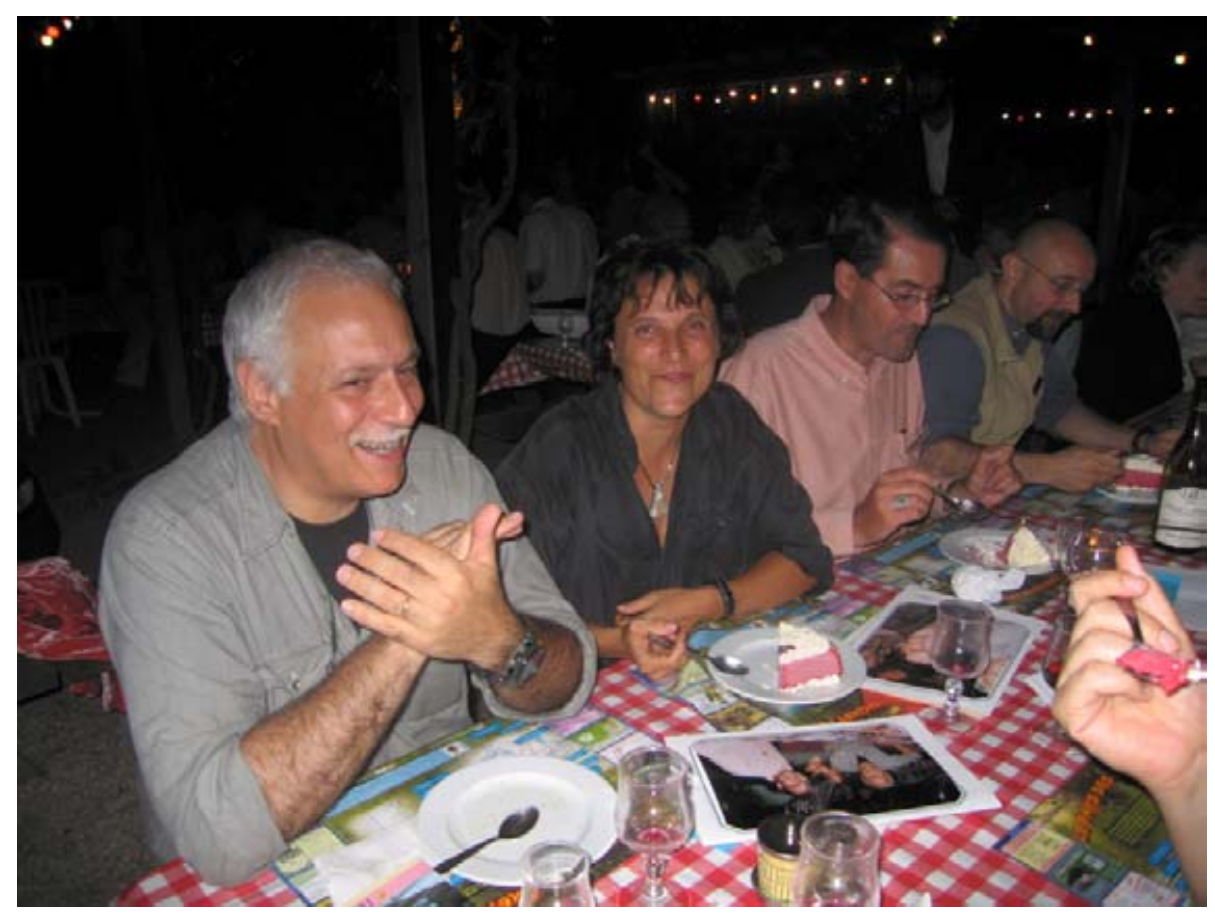

Alessandro Chieffi, Livia Origlia, Claus Leitherer and Marco Limongi. 


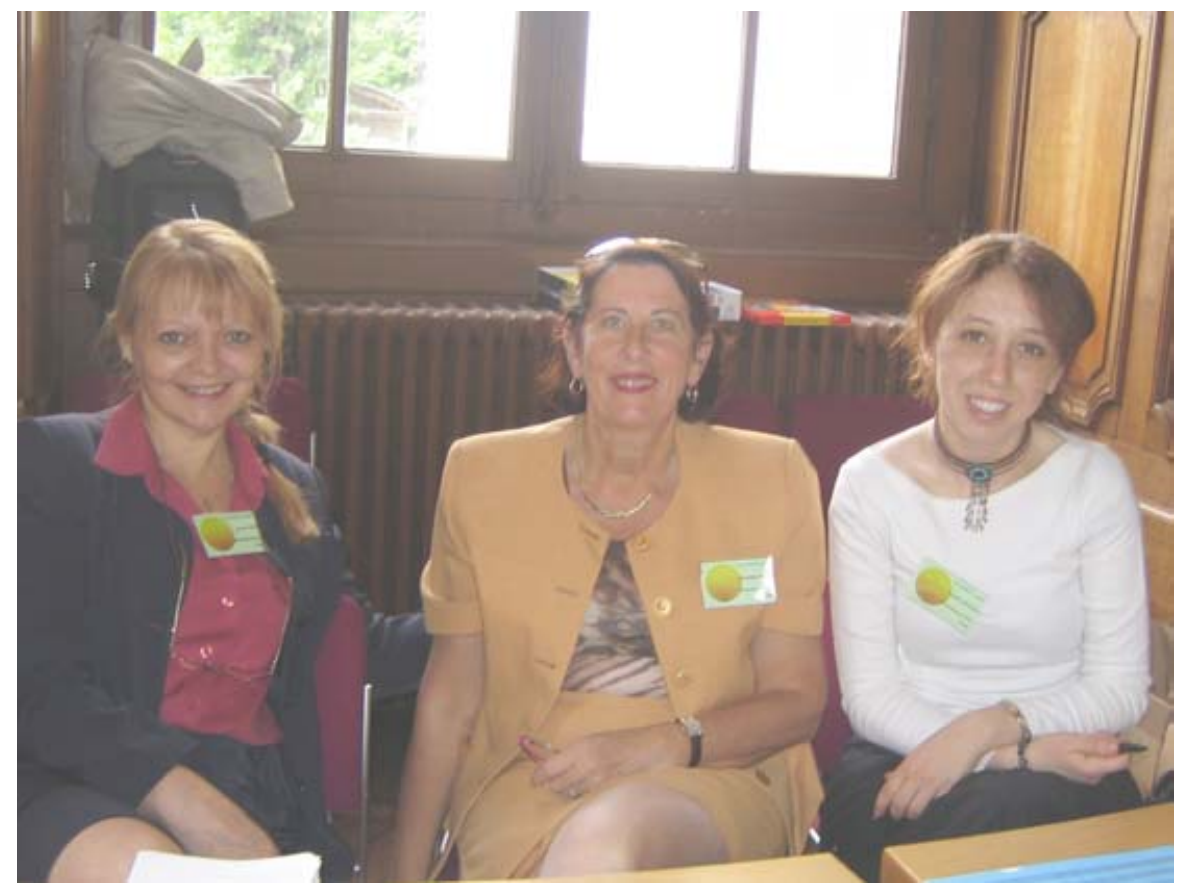

Annick Oger, Jacqueline Pluet and Cherifa Zouaoui, the administration team of the conference.

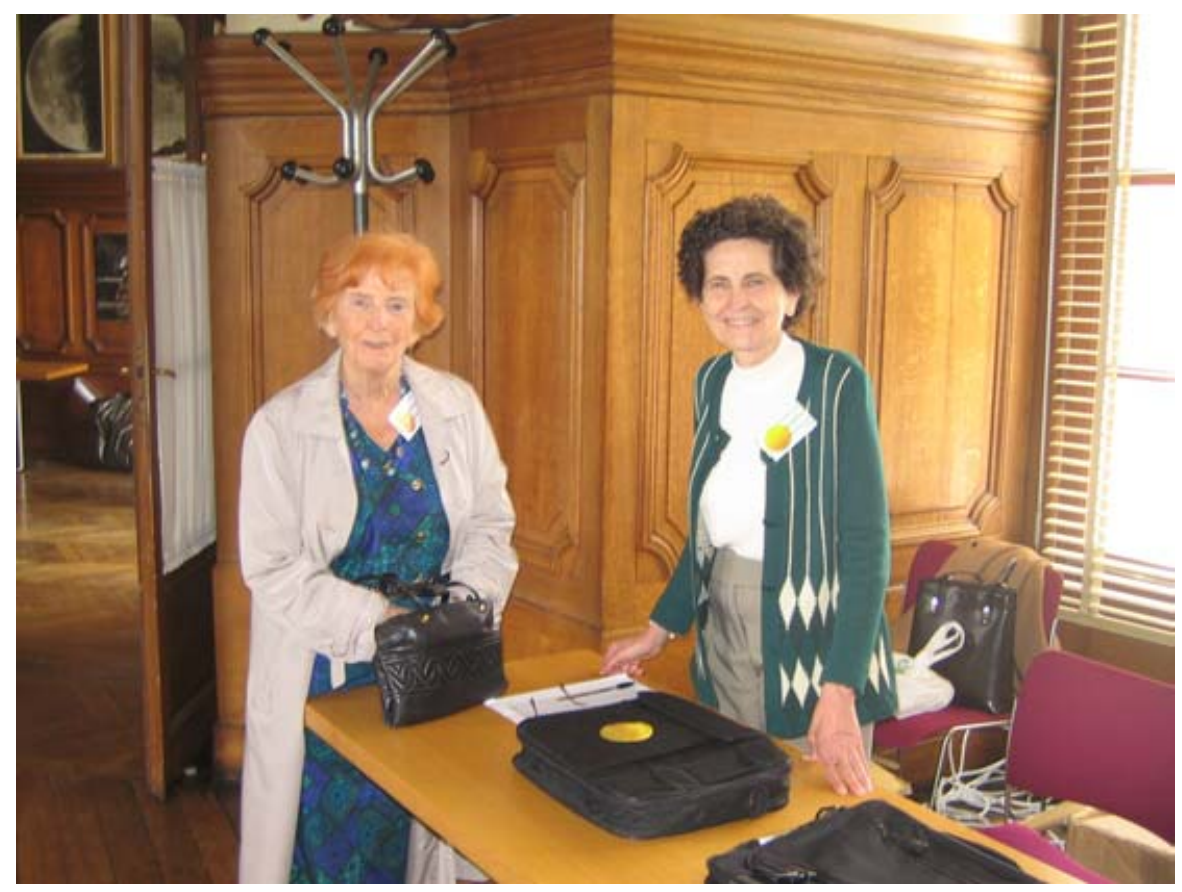

Giusa Cayrel and Claire Bentolila preparing to distribute the conference bags to participants at the welcome reception. 